\title{
N-Glycan Modification in Covid-19 Pathophysiology: In vitro Structural Changes with Limited Functional Effects
}

\author{
Cristiane J. Nunes-Santos ${ }^{1} \cdot$ Hye Sun Kuehn ${ }^{1} \cdot$ Sergio D. Rosenzweig ${ }^{1}$ (D) \\ Received: 20 August 2020 / Accepted: 28 October 2020 / Published online: 27 November 2020 \\ (C) This is a U.S. Government work and not under copyright protection in the US; foreign copyright protection may apply 2020
}

\begin{abstract}
In 2014, we reported two siblings with a rare congenital disorder of glycosylation due to mutations in mannosyl-oligosaccharide glucosidase (MOGS). The glycan alteration derived from this disease resulted in an in vitro infection resistance to particular enveloped, N-glycosylation-dependent viruses as influenza and HIV. As part of the global effort to find safe and effective antiviral therapies for Covid-19, we assessed the in vitro activity of the FDA-approved $\alpha$-glucosidase inhibitor miglustat against SARS-CoV-2. Expression plasmids encoding SARS-CoV-2 spike (S) and human ACE2 glycoproteins (GP) were tested to evaluate $\mathrm{N}$-glycan modifications induced by $\alpha$-glucosidase inhibition. Immunoprecipitation was used to assess binding between these two GP. Cell-to-cell fusion was assessed by immunofluorescence of cocultures of SARS-CoV-2 S and ACE2-expressing cells. Miglustat effect on immune response was tested by measuring cytokine release from PBMC exposed to purified SARSCoV-2 S. In our overexpression system, miglustat successfully and specifically modified N-glycans in both SARS-CoV-2 S and its main receptor ACE2. Binding between these two GP was not affected by glycan modifications. A surrogate marker for viral cytopathic effect, measured as receptor-dependent SARS-CoV-2 S-driven cell-to-cell fusion, was not disrupted by miglustat treatment. This observation was further confirmed in MOGS-null transfected cells. Miglustat produced no statistically significant effects on cytokine production following SARS-CoV-2 S glycoprotein stimulation of PBMC. Our work shows that despite clear $\mathrm{N}$-glycan alteration in the presence of miglustat, the functions of the Covid-19-related glycoproteins studied were not affected, making it unlikely that miglustat can change the natural course of the disease.
\end{abstract}

Keywords Glycosylation $\cdot \alpha$-glucosidase $\cdot$ miglustat $\cdot$ SARS-CoV-2 $\cdot$ spike $\cdot$ ACE2

\section{Introduction}

In 2014, we reported two siblings with a rare congenital disorder of glycosylation (CDG) due to mutations in mannosyloligosaccharide glucosidase (MOGS) [1]. These patients were resistant to infections by particular, enveloped viruses with $\mathrm{N}$ glycosylated glycoproteins. Lessons learned from these siblings, and previous attempts to manipulate MOGS, highlight the reliance of particular viruses on the host $\mathrm{N}$-glycosylation machinery and a vulnerability that can be exploited in favor of

Cristiane J. Nunes-Santos and Hye Sun Kuehn contributed equally to this work.

Sergio D. Rosenzweig

srosenzweig@cc.nih.gov

1 Immunology Service, Department of Laboratory Medicine, National Institutes of Health (NIH) Clinical Center, 10 Center Dr., Bldg 10, Rm. 2C410F, Bethesda, MD 20892, USA the host [2]. MOGS (or $\alpha$-glucosidase 1) is the first enzyme in the N-linked glycosylation trimming pathway. MOGS acts in the endoplasmic reticulum (ER) and is responsible for removing the terminal $\alpha 1-2$ glucose from the 14 sugars basic $\mathrm{N}$ glycan moiety ( 3 glucose, 9 mannose, and $2 \mathrm{~N}$ acetylglucosamine-- $\mathrm{Glc}_{3} \mathrm{Man}_{9} \mathrm{GlcNac}_{2-}$ ) that initiates the glycoprotein folding quality control process [3]. MOGS defects or manipulation alters this process resulting in untrimmed, bulkier, N-glycans that differ from the MOGS wild-type/unmanipulated-produced glycoprotein of interest. This effect impacts both human (as viral receptors) and virus-encoded glycoproteins (as gp120 for the human immunodeficiency virus-HIV) [4]. The glycan alteration seen in the MOGSCDG patients resulted in an in vitro resistance to enveloped, $\mathrm{N}$-glycosylated viruses such as HIV and influenza, but not to non-enveloped or non-N-glycosylation-dependent viruses such as Hepatitis A, Adenovirus, or Vaccinia virus [1].

The emergence of a novel rapidly spreading virus in China, severe acute respiratory syndrome coronavirus 2 (SARS- 
CoV-2) in December 2019, led to a pandemic within a short span of time [5, 6]. Coronavirus disease 2019 (Covid-19) has a broad clinical spectrum, not yet fully described or understood, with a concerning potential for severe respiratory disease, multiorgan involvement, and death $[7,8]$. Because containment of the virus has proven to be extremely difficult, mitigation efforts such as mask-wearing, physical distancing, confinement, and quarantines have been implemented worldwide resulting in limited exposures/contagious events [9] with also a strong social, health, and economic burden $[10,11]$. Since ideal preventive strategies such as vaccines have an inevitably long development, testing, and manufacturing time [12], repurposing of known drugs to treat Covid-19 emerges as an attractive approach to timely fulfill the ongoing need.

Among several potential therapeutic targets, great attention has been directed to the coronavirus spike (S) glycoprotein. This heavily glycosylated protein (22 potential N-linked glycosylation sites) forms homotrimers on the surface of the viral envelope creating spikes that confer the crown-shaped aspect which originated the virus family name [13, 14]. The presence of a furin cleavage site on the spike glycoprotein of SARSCoV-2 is a distinct feature from other closely related coronaviruses [15]. The S glycoprotein is critical for two key steps in the viral life cycle: binding of the virion to the target cell receptor and fusion of viral and cellular membranes $[13,16]$.

Pharmacological inhibition of ER $\alpha$-glucosidases with sugar mimetics (iminosugars) has been proposed as an antiviral therapy for enveloped/ $\mathrm{N}$-glycosylation-dependent viral infections $[2,17,18]$. Host-targeting antivirals are less likely to lose efficacy in the scenario of viral mutations. Moreover, many viral receptors, including human angiotensin-converting enzyme 2 (ACE2), the main receptor for SARS-CoV-2, are heavily $\mathrm{N}$-glycosylated themselves and also potential targets of the therapy [19].

In this study, we assessed the in vitro activity of miglustat (N-butyldeoxynojirimycin, NB-DNJ) in Covid-19 pathophysiology. Miglustat was originally developed as an anti-HIV drug based on its $\alpha$-glucosidase inhibitory effect [20]; later, the drug was FDA approved for the treatment of NiemannPick disease type $\mathrm{C}$ and Gaucher disease because of its glucosylceramide synthase inhibitor activity [21, 22]. Considering the extensive N-glycosylation of both proteins, the SARS-CoV-2 envelope Spike protein and its main human receptor ACE2 were targeted in our work.

\section{Methods}

Peripheral Blood Mononuclear Cells Blood from healthy donors were obtained under approved protocols by the National Institutes of Health institutional review board. All procedures were based on standard of care and established clinical guidelines were followed. Peripheral blood mononuclear cells (PBMC) were isolated from whole blood via density gradient centrifugation using Ficoll-Paque Plus (GE healthcare).

Cell Culture Human embryonic kidney 293 T (HEK293T) cells (ATCC®; CRL-3216), NIH3T3 cells (ATCC®; CRL1658), and primary fibroblasts from a normal control and a MOGS-deficient patient (previously reported - patient 2 from [1] with compound heterozygous mutations in $M O G S$ ) were cultured in Dulbecco's Modified Eagle's Medium (DMEM, Gibco) supplemented with $10 \%$ fetal bovine serum, 2 mM Lglutamine, $100 \mathrm{U} / \mathrm{mL}$ penicillin, and $100 \mu \mathrm{g} / \mathrm{mL}$ streptomycin (Gibco) at $37{ }^{\circ} \mathrm{C}$ in a humidified $5 \% \mathrm{CO}_{2}$ incubator.

Expression Plasmids and Purified Protein Codon-optimized expression plasmids used in this study were transfected into HEK293T cells using Effectene ${ }^{\circledR}$ Transfection Reagent (Qiagen) following manufacturer's protocol. pCMV3-2019nCoV-Spike (S1 + S2)-long-HA (Cat. VG40589-CY) and pCMV3-ACE2-Flag (Cat. HG10108-CF) were purchased from Sino Biological Inc. Purified recombinant SARS-CoV2 Spike S1+S2 ECD-His (Cat. 40589-V08B1) was purchased from Sino Biological.

Iminosugars and Ceramide Analog N-Butyldeoxynojirimycin (NB-DNJ or miglustat; solubilized in water) was a gift from Emergent BioSolutions. Castanospermine (solubilized in DMSO) was purchased from Santa Cruz Biotechnology (Cat. sc-201358). Specific glucosylceramide inhibitor eliglustat (solubilized in DMSO) was purchased from Selleckchem (Cat. S7852).

Enzymatic Digestion Peptide-N-glycosidase F (PNGase F) and endoglycosidase $\mathrm{H}$ (Endo H) (New England Biolabs Inc., Cat. P0704 and P0702) were used for complete and partial removal of N-linked oligosaccharides, respectively. Manufacturer's instructions were followed. Briefly, 10 $20 \mu \mathrm{g}$ of protein lysates were mixed with glycoprotein denaturing buffer and heated at $100{ }^{\circ} \mathrm{C}$ for $10 \mathrm{~min}$. Denatured proteins were cooled down on ice, centrifuged, and mixed with Glycobuffer 2, $\mathrm{H}_{2} \mathrm{O}$, and 10\% NP-40 (PNGase F reaction) or Glycobuffer 3 and $\mathrm{H}_{2} \mathrm{O}$ (Endo $\mathrm{H}$ reaction). The specific endoglycosidase was added to each reaction and samples were incubated at $37^{\circ} \mathrm{C}$ for $1 \mathrm{~h}$. Reaction products were further analyzed by Western blotting as described below.

Western Blotting and Immunoprecipitation For total cell extracts, cells were washed with PBS once and lysed in lysis buffer (50 mM Tris pH 7.4, $150 \mathrm{mM} \mathrm{NaCl}, 2 \mathrm{mM}$ EDTA, $0.5 \%$ Triton X-100, and $0.5 \%$ NP40 and halt protease and phosphatase inhibitor cocktail [Sigma, Cat. PPC1010]) and samples were adjusted to have equal concentration of total protein and subjected to immunoblotting. For the 
immunoprecipitation, cell lysates were prepared in IP buffer (50 mM Tris pH 7.4, $150 \mathrm{mM} \mathrm{NaCl}, 2 \mathrm{mM}$ EDTA, $0.5 \%$ Triton X-100, or with $0.5 \%$ NP-40 and halt protease and phosphatase inhibitor cocktail). Total protein $(500 \mu \mathrm{g})$ was incubated with an anti-FLAG antibody (Cell signaling technology, Cat. 14793) and $40 \mu \mathrm{l}$ Protein A/G-agarose beads (Pierce). After $2-4 \mathrm{~h}$ incubation at $4{ }^{\circ} \mathrm{C}$ on a rotating wheel, beads were washed three times with IP buffer; samples were prepared and separated on a NuPAGE® Novex ${ }^{\circledR} 4-12 \%$ Bis-Tris Protein Gels (Life Technology) and transferred to nitrocellulose membrane using Trans-Blot Turbo Transfer system according to the manufactural instruction (Bio-Rad). The membranes were incubated with antibodies to SARS-CoV-2 S glycoprotein (GeneTex, Cat. GTX632604, recognizing an epitope within the S2 subunit), human ACE2 (Cell Signaling Technology, Cat. 4355), anti-FLAG antibody (Cell signaling technology, Cat. 14793), or $\beta$-actin (Cell Signaling Technology, Cat. 4970). Membranes were then washed and incubated with a secondary antibody labeled with HRP (Jackson Immuno Research, 111-035-003 or 115-035-003). The Western blot images were acquired with C-Digit scanner using Image Studio Software (Li-Cor).

Cell-Cell Fusion Assay NIH3T3 cells were seeded at $4 \times 10^{4}$ on a 12-well plate and independently transfected with pCMV32019-nCoV-Spike (S1 + S2)-long-HA or pCMV3-ACE2Flag using Effectene $®$ Transfection Reagent (Qiagen) following manufacturer's protocol. Transfection efficiency was similar for both vectors at 20-30\% (not shown). On the following day, $1 \mathrm{mM}$ miglustat was added to transfected cells when indicated. After $24 \mathrm{~h}$, cells were trypsinized and counted, and SARS-CoV-2 S transfected cells were cocultured at 1:1 ratio with ACE2 transfected cells (miglustat treatment was maintained in treated cocultures). Primary fibroblasts were transfected with the same plasmids using Amaxa ${ }^{\circledR}$ Human Dermal Fibroblast Nucleofector ${ }^{\circledR}$ kit, program U-023, following manufacturer's instructions and cocultured at a 1:1 ratio. After $48 \mathrm{~h}$, cells were washed twice in PBS, fixed for $15 \mathrm{~min}$ in $4 \%$ paraformaldehyde and permeabilized for $15 \mathrm{~min}$ in $0.1 \%$ Triton X-100 in PBS at room temperature, and incubated for $30 \mathrm{~min}$ in blocking buffer (1\% FBS and $0.1 \%$ Triton X100 in PBS). Cells were incubated for $2 \mathrm{~h}$ with mouse anti-HA antibody and rabbit anti-Flag antibody in blocking buffer (Cell signaling technology, Cat. 2367 and 14793). Cells were then washed twice with PBS and incubated for $1 \mathrm{~h}$ with Alexa Fluor 488 (green color) and Alexa Fluor 594 (red color)-conjugated secondary antibodies in blocking buffer (Invitrogen, Cat. A-11001 and A-11072). Next, cells were washed twice with PBS and incubated with DAPI (Cell Signaling technology, Cat. 4083S) in PBS for 10 min, followed by 2 additional washes with PBS. Representative images (range, 8-20) per each condition from 2 independent experiments were collected from NIH3T3 and primary fibroblast cocultures, respectively, in a ZOE fluorescent cell imager (Bio-Rad) and analyzed in ImageJ software (National Institutes of Health).

Cytokine Analysis Total PBMC (100,000 cells/100 $\mu$ l) were preincubated with or without $1 \mathrm{mM}$ miglustat for $24 \mathrm{~h}$, followed by $5 \mu \mathrm{g}$ of purified SARS-CoV-2 S protein for an additional $24 \mathrm{~h}$. Cell-free supernatants were harvested, and cytokines were measured simultaneously with Human XL Cytokine Luminex Performance Panel (R\&D Systems) using the Luminex 200 System (Luminex Corporation).

Statistical Analysis Statistical analyses were performed using GraphPad Prism software version 8.3.0 (GraphPad, LLC). P values $<0.05$ were considered statistically significant.

\section{Results}

Expression and N-Glycosylation Pattern of SARS-CoV-2 S and Human ACE2 Glycoproteins To test glycosylation patterns of SARS-CoV-2 and its receptor ACE2, each protein was overexpressed on HEK293T cells and modification of glycosylation was evaluated by immunoblotting after treatment with PNGase F or Endo H. While PNGase F cleaves all Nglycans, Endo H cleaves only the high-mannose and hybrid branches of $\mathrm{N}$-glycans and discriminates between $\mathrm{N}$ glycosylated glycoproteins that did not egress the ER or completed its trimming process (Endo $\mathrm{H}$ sensitive) and those that have egressed the ER and completed the trimming process (Endo H resistant). Undigested lysates from SARS-CoV-2 S transfected cells showed two bands on Western blot analysis (Fig. 1a), the higher molecular weight band $(\sim 180 \mathrm{kDa})$ corresponding to the full-length SARS-CoV-2 S glycoprotein and a lower molecular weight one $(\sim 90 \mathrm{kDa})$ consistent with the SARS-CoV-2 S2 subunit, demonstrating efficient expression and proteolytic processing in the overexpression system. One main band at the expected molecular weight for glycosylated ACE2 $(\sim 120-135 \mathrm{kDa})$ was detected in the undigested lysate from ACE2 transfected cells (Fig. 1b). PNGase F treatment resulted in a mobility shift for both SARS-CoV-2 S and ACE2 bands indicating the presence of N-linked glycosylation (Fig. 1a and b). Endo H-treated samples displayed two bands with different migration patterns: a faster Endo Hsensitive band and a slower Endo H-resistant band. The Endo $\mathrm{H}$-resistant band was stronger in ACE2 expressing cells demonstrating a higher content of mature Golgi/post-ER forms or complex-type N-glycans. A predominance of complex-type $\mathrm{N}$-glycans on human ACE2 has been observed before [23, 24].

Miglustat Modified the N-Glycosylation Pattern of SARS-CoV$2 \mathrm{~S}$ and Human ACE2 Glycoproteins Transfection of HEK293T cells with SARS-CoV-2 or human ACE2 plasmids 
a

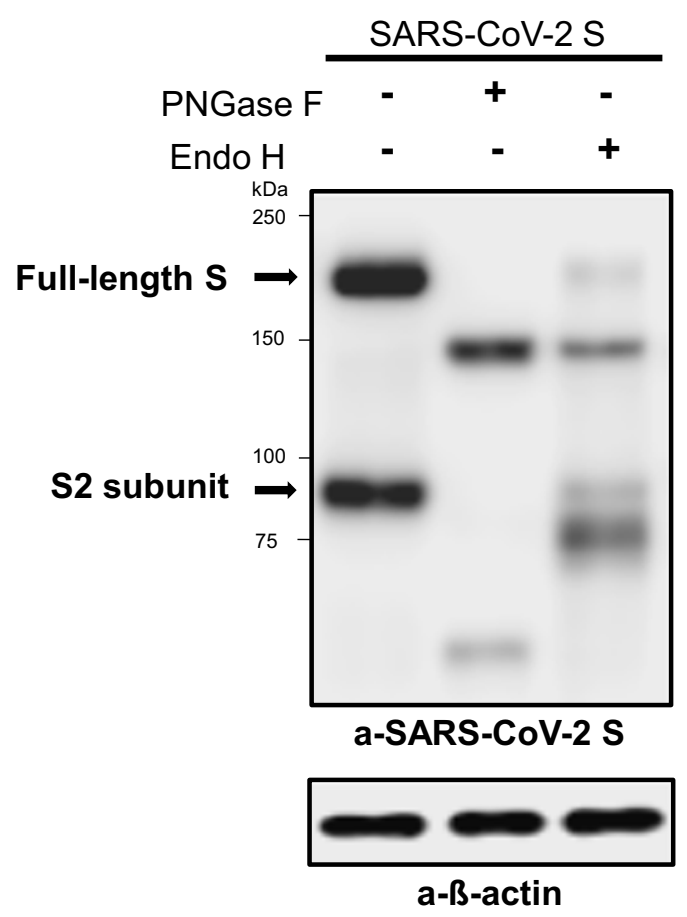

Fig. 1 Expression and enzymatic digestion of SARS-CoV-2 S and human ACE2 in transfected HEK 293T cells. HEK293T cells were transfected with SARS-CoV-2 S (a) or ACE2 (b). On the following day, cell lysates were digested with PNGase F (lane 2) or Endo H (lane

followed by treatment with the $\alpha$-glucosidase inhibitors castanospermine and miglustat resulted in detection of glycoprotein bands at a higher molecular weight on treated lysates compared to untreated controls or samples treated with a specific glucosylceramide synthase inhibitor (eliglustat) in both SARS-CoV-2 and ACE2 Western blots (Fig. 2a and b). This result was consistent with the specific inhibition of MOGS and the $\alpha$-glucosidase $\mathrm{N}$-glycan trimming process in the ER

a

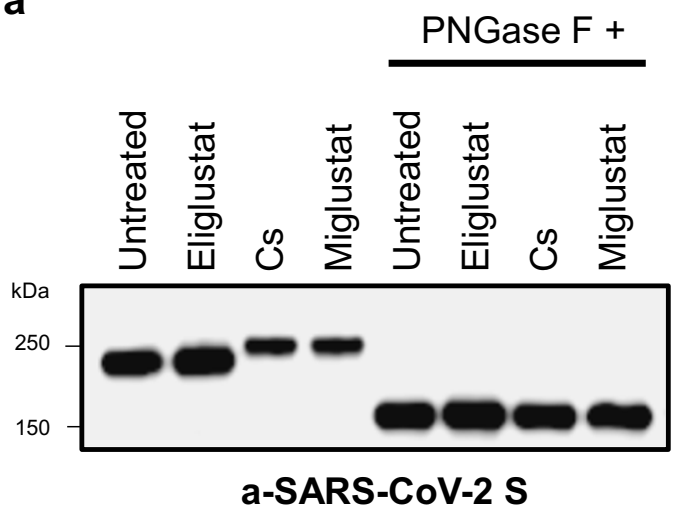

Fig. 2 Miglustat treatment of SARS-CoV-2 S and human ACE2 expressing HEK293T cells. HEK293 cells were transfected with SARS-CoV-2 $\mathrm{S}$ (a) or ACE2 (b). On the next day, iminosugar treatment (miglustat $1 \mathrm{mM}$ or castanospermine $1 \mathrm{mM}$ ) or glucosylceramide synthase inhibitor (eliglustat $10 \mu \mathrm{M}$ ) was started. After $24 \mathrm{~h}$, cells were lysed and half of the b

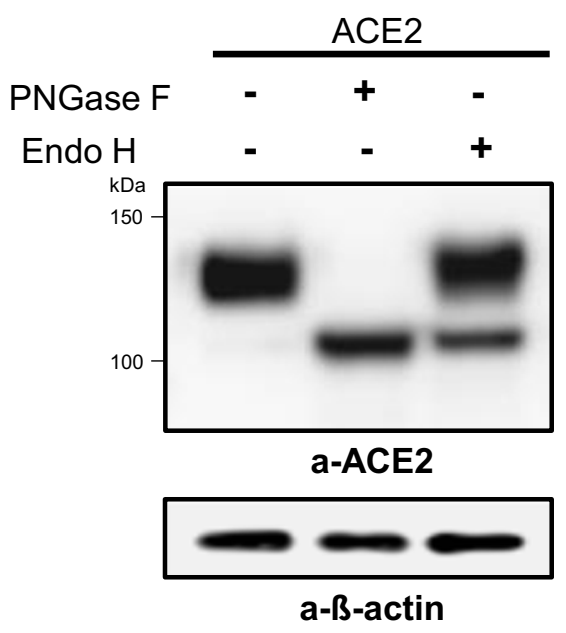

3) and immunoblotted with anti-SARS-CoV-2 S antibody specific for the $\mathrm{S} 2$ subunit of the S protein or anti-ACE2 antibody, as indicated. $B$-actin was used as a loading control. Data shown are representative of 2 independent experiments

by miglustat, with no evidence of glucosylceramide synthase inhibition effect in this function. To further explore the protein size difference induced by $\alpha$-glucosidase inhibition, we subjected original cell lysates from untreated and treated samples to PNGase F digestion and repeated immunoblotting. Enzymatic removal of $\mathrm{N}$-glycans abolished the size difference between treated samples and untreated controls in both expression systems showing that $\mathrm{N}$-glycan modifications were

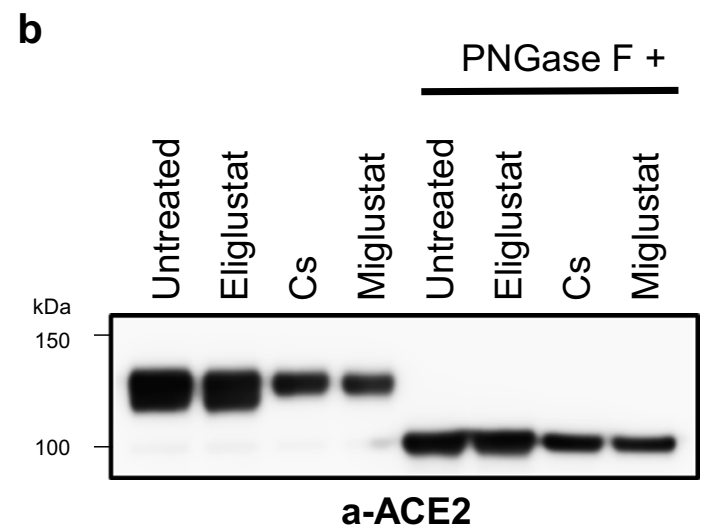

lysates were digested with PNGase F for $1 \mathrm{~h}$. Protein lysates before and after PNGase F digestion were separated by Western blotting with antiSARS-CoV-2 S antibody and anti-ACE2 antibody as indicated. Cs = castanospermine. Data shown are representative of 2 independent experiments 
responsible for the difference in migration pattern observed upon $\alpha$-glucosidase inhibition (Fig. $2 \mathrm{a}$ and b).

\section{Binding of SARS-CoV-2 S to ACE2 Receptor Was Not Affected} by Miglustat Treatment To investigate whether glycan modifications caused by miglustat treatment could disrupt binding of SARS-CoV-2 S glycoprotein to its main receptor ACE2, immunoprecipitation (IP) assays were performed. First, to independently explore the effect of glycan modification of host glycoproteins, HEK293T cells were transfected with ACE2-Flag and treated with $\alpha$-glucosidase inhibitors miglustat or castanospermine. On the next day, total cell lysates were prepared and mixed with purified recombinant SARS-CoV-2 S glycoprotein followed by IP with anti-Flag antibody and immunoblotting with anti-SARS-CoV-2 S antibody. Neither miglustat nor castanospermine pretreatment of ACE2 expressing cells prevented the binding of SARS-CoV-2 S protein to ACE2 (Fig. 3a). We then assessed the effect of miglustat on HEK293T cells cotransfected with ACE2-Flag and SARS-CoV-2 S-HA protein to allow simultaneous modification of viral and host glycoproteins. Despite successful altering of spike protein glycosylation (detected by a mobility shift of treated samples on Western blot analysis), binding to

a

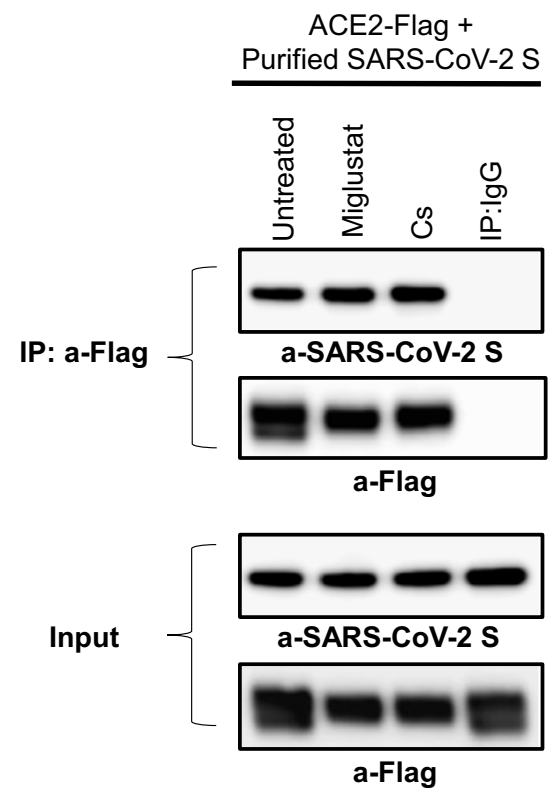

Fig. 3 Effect of miglustat treatment on SARS-CoV-2 S to ACE2 binding. (a) HEK293T cells were transfected with Flag-tagged ACE2 for $24 \mathrm{~h}$, followed by indicated inhibitor treatment for an additional $24 \mathrm{~h}$. Protein extracts were prepared in lysis buffer containing $0.5 \%$ Triton X-100 and mixed with $5 \mu \mathrm{g}$ of SARS-CoV-2 S protein. Same amount of untreated and treated protein lysates was immunoprecipitated with anti-Flag antibodies and probed with anti-SARS-CoV-2 S and anti-Flag antibodies. (b) HEK293T cells were transfected with Flag-tagged ACE2 together with
ACE2 remained unchanged after miglustat treatment in the IP assay (Fig. 3b). We also tested the hypothesis that glycosylation modifications could alter binding avidity/affinity of SARS-CoV-2 S to ACE2 receptor by using different gradient of sodium dodecylsulfate (SDS), a strong anionic detergent, on HEK293T cells cotransfected with Flag-ACE2 and HA-SARS-CoV-2 S cultured in the presence or absence of miglustat. Our results demonstrated that miglustat treatment did not change their binding neither in the milder nonionic detergent (NP-40 and Triton X-100; non-denaturing) nor in the harsh denaturing ionic detergent conditions, suggesting that the interaction between SARS-CoV-2 S protein and ACE2 did not appear to be affected by structural N-glycosylation changes or different affinity/ avidity environmental conditions (Fig. 4).

Receptor-Dependent Cell-to-Cell Fusion Mediated by SARSCoV-2 S Was Not Inhibited by ER a-Glucosidase Inhibition We assessed the effect of ER $\alpha$-glucosidase inhibition in SARS-CoV-2 S glycoprotein-driven cell-to-cell fusion by evaluating syncytia/multinucleated giant cell formation in cocultures of NIH3T3 cells independently transfected with SARS-CoV-2 S-HA or ACE2-Flag in the presence or absence of miglustat. Syncytia formation was assessed by

b

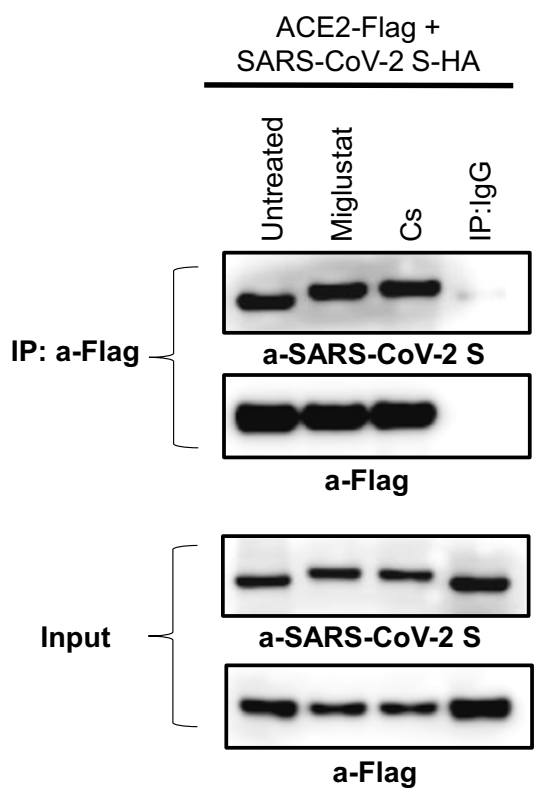

HA-tagged SARS-CoV-2 S for $24 \mathrm{~h}$, followed by indicated inhibitor treatment for an additional $24 \mathrm{~h}$. Protein extracts were prepared in lysis buffer containing $0.5 \%$ Triton X-100 and immunoprecipitated with antiFlag antibodies and probed with anti-SARS-CoV-2 S and anti-Flag antibodies. Two percent of the total volumes of the whole cellular lysates used for IP reactions were loaded as input controls. Cs $=$ castanospermine. Data shown are representative of 3 independent experiments 


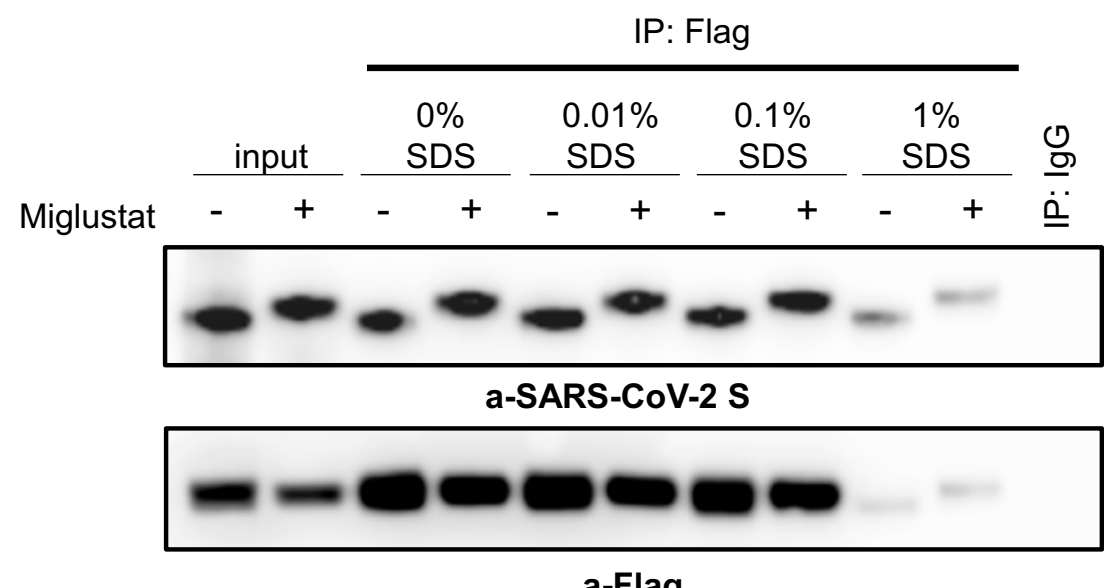

Fig. 4 Effect of miglustat treatment on SARS-CoV-2 S to human ACE2 binding affinity. HEK293T cells were transfected with Flag-tagged ACE2 together with HA-tagged SARS-CoV-2 S for $24 \mathrm{~h}$, followed by $1 \mathrm{mM}$ miglustat treatment for an additional $24 \mathrm{~h}$. Protein extracts were

immunofluorescence. No statistically significant differences were observed in syncytia formation frequency or size comparing miglustat-treated samples to untreated controls (Fig. 5a and $b$ ). We further investigated the effect of null $\alpha$ glucosidase activity on SARS-CoV-2 S glycoprotein-driven cell-to-cell fusion by applying a similar protocol to primary

\section{a-Flag}

prepared in lysis buffer containing $0.5 \%$ Triton X-100 and $0.5 \%$ NP-40. The same amount of protein lysates was divided and indicated percentage of SDS was added in the lysates and performed immunoprecipitation. Data shown are representative of 3 independent experiments

fibroblasts from a MOGS-null patient in comparison to a normal control. Immunofluorescence analysis from cocultured cells showed that the patient's cells formed multinucleated giant cells at a similar frequency as the normal control cells. On an individual syncytia composition analysis, more nuclei were detected on the MOGS-null patient's multinucleated a
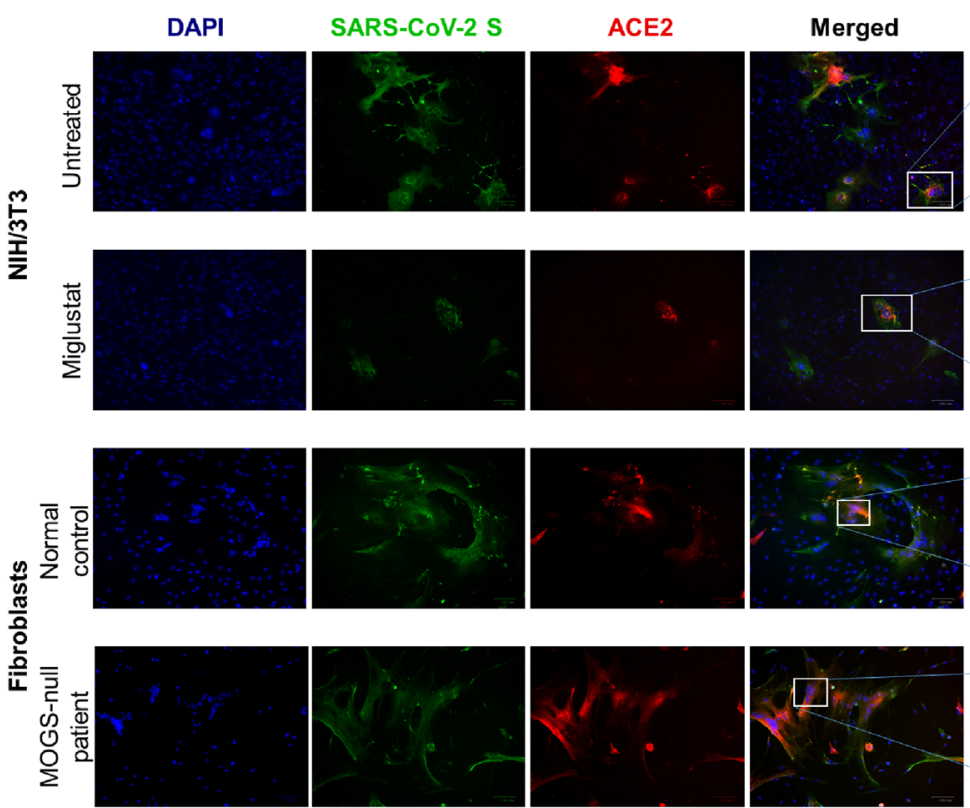

b
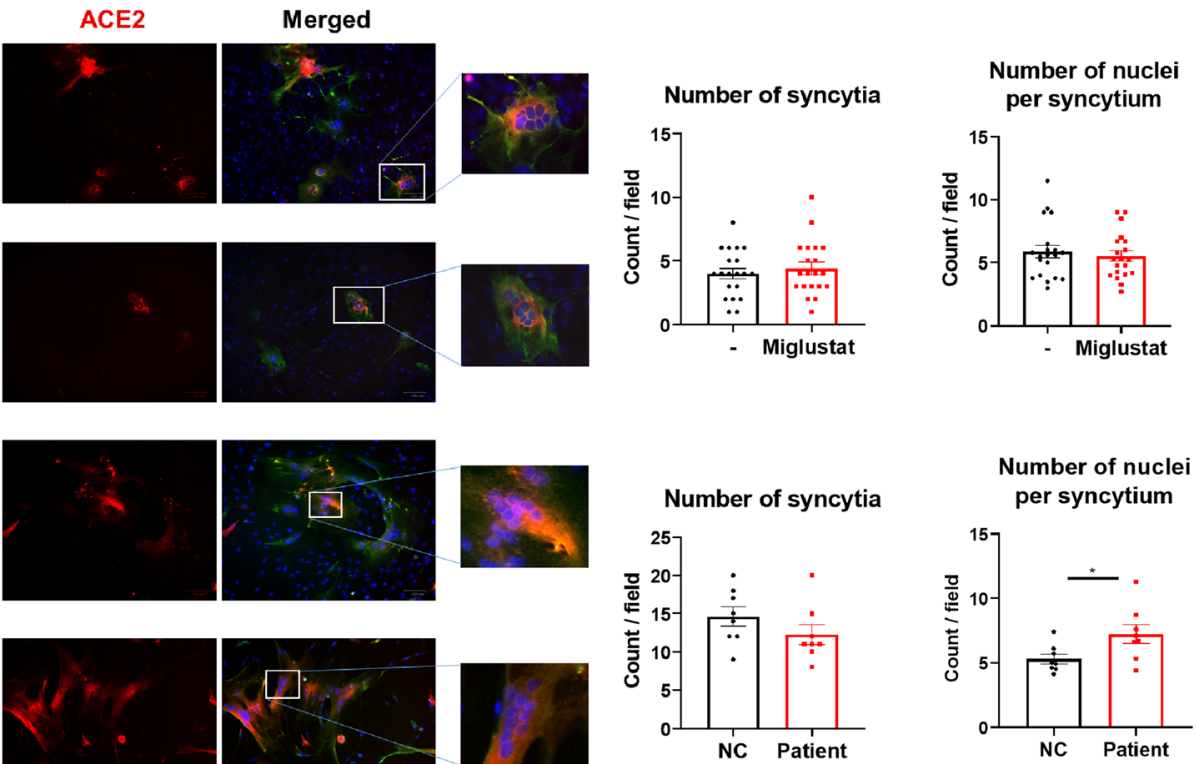

Fig. 5 Effect of ER $\alpha$-glucosidase inhibition on syncytia formation between SARS-CoV-2 S and human ACE2 transfected cells. (a) NIH/3T3 cells (upper panels) transfected with SARS-CoV-2 S-HA or ACE2-Flag were cultured in the presence or absence of $1 \mathrm{mM}$ miglustat. Primary fibroblasts (bottom panels) from a MOGS-null patient or a normal control were transfected with the same plasmids. SARS-CoV-2 S transfected cells were mixed at 1:1 ratio with ACE2 transfected cells and cocultured for $48 \mathrm{~h}$. Cells were then fixed, permeabilized, and labeled with mouse
anti-HA antibody and rabbit anti-Flag antibody followed by Alexa Fluor 488 (green) anti-mouse and Alexa Fluor 594 (red) anti-rabbit secondary antibodies. DAPI was used for nuclei staining. Images are representative of multinucleated giant cells (syncytia) formation from 2 independent experiments (original magnification $\times 175$ ). (b) Quantitative comparison of syncytia formation between miglustat-treated samples and untreated controls (top row) or normal control (NC) and MOGS-null patient (bottom row). $* p<0.05$, student $t$ test. Data are mean \pm SEM 
giant cells when compared with the normal control cells (Fig. $5 \mathrm{a}$ and $\mathrm{b})$.

SARS-CoV-2 S Glycoprotein-Induced Cytokine Generation in PBMC Was Not Affected by Miglustat To evaluate the effect of miglustat on cytokine secretion, PBMC from normal controls were incubated with purified SARS-CoV-2 S glycoprotein with/without miglustat pretreatment and MCP-1, MIP-1a, IL-10, IL-6, TNF $\alpha$, and IL- 8 were evaluated in the supernatants. Miglustat produced no statistically significant effects on cytokine production following SARS-CoV-2 S glycoprotein stimulation of PBMC (Fig. 6).

\section{Discussion}

The Covid-19 pandemic has created a pressing need for an effective and safe treatment for SARS-CoV-2 infection with the challenge of minimum research time. Repurposing of drugs presenting proven or potential antiviral activity represents one of the quickest routes to achieve this goal, taking advantage of their characterized safety profile, albeit in the context of different indications.
Manipulating the host glycosylation machinery by inhibiting ER $\alpha$-glucosidases, a broad-spectrum antiviral strategy validated by MOGS-CDG patients, seemed an attractive approach in the face of a novel virus which mutation rate is still unclear. We chose miglustat as a candidate because of its regulatory status (FDA approved), safety profile [25], and previous in vitro data supporting beneficial effects against severe acute respiratory syndrome coronavirus (SARS-CoV) [18, 26, 27].

Studies from SARS-CoV highlighted the ability of the virus to recognize its receptor as one of the strongest determinants of infectivity and transmissibility [28]. Extensive work has been dedicated to characterize the binding interface of SARS-CoV-2 S protein and its functional receptor ACE2 $[19,29-31]$. N-linked glycosylation sites in close relation to binding regions are present in both proteins [14, 23]. The functional impact of glycan manipulation on the viral-host interaction, however, can be largely unpredictable. For instance, Li et al. [32] reported reduced infectivity of SARSCoV-2 after deletion of glycosylation sites on the spike protein, while Mehdipour et al. [33] (preprint) performing molecular dynamics simulations found that glycosylation of one specific site on ACE2 (N90) weakened binding to SARSCoV-2 S protein. Likewise, deep mutagenesis analysis

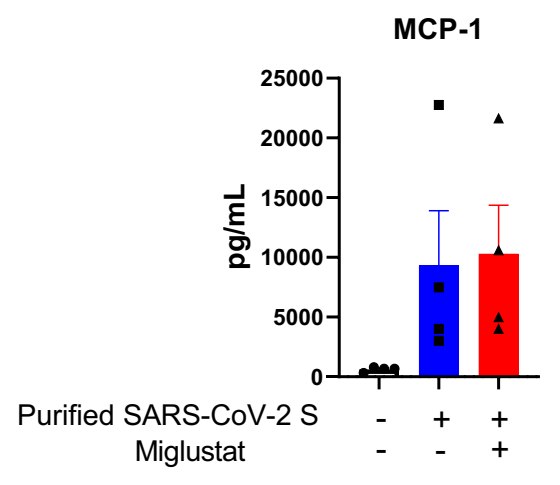

IL-6

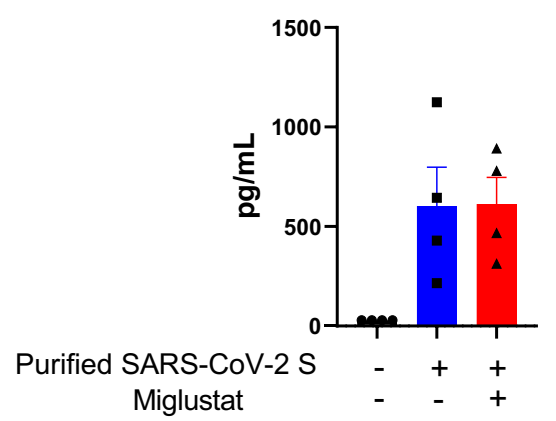

Fig. 6 Cytokine release induced by SARS-CoV-2 S glycoprotein in the presence or absence of miglustat. Peripheral blood mononuclear cells (PBMC) from 4 normal controls were cultured in the presence (red bars) or absence (blue bars) of miglustat at $1 \mathrm{mM}$ for $24 \mathrm{~h}$. Then, purified SARS-CoV-2 S protein was added to the cultures for an additional
MIP-1a

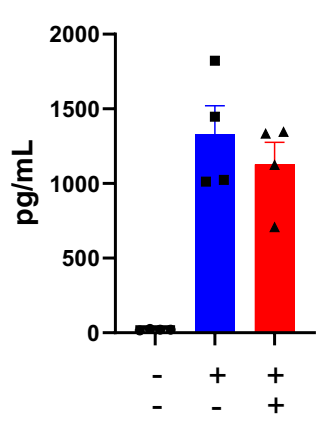

TNF $\alpha$

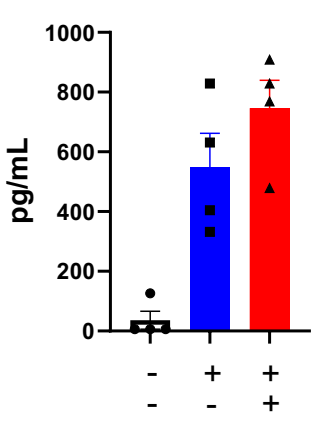

IL-10

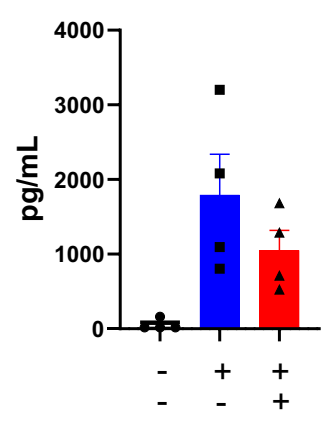

IL-8

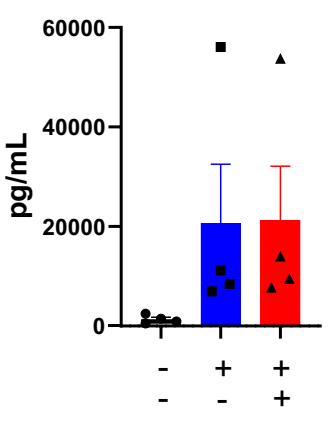

$24 \mathrm{~h}$. Untreated, unstimulated samples are presented as baseline controls. Cytokine levels were measured in culture supernatants using Luminex assay. Differences between groups were not statistically significant ( $p>0.05$,Wilcoxon signed-ranked test). Data shown are representative of 2 independent experiments and are expressed as mean \pm SEM 
reported in another preprint [34] revealed that several mutants affecting the N90 glycosylation motif of ACE2 resulted in enhanced binding to SARS-CoV-2 $\mathrm{S}$ receptor binding domain. In our study, $\mathrm{N}$-glycan modifications of ACE2 receptor alone or in combination with SARS-CoV-2 S protein induced by miglustat treatment could not abrogate or reduce binding affinity between these two proteins. Similarly, Zhao et al. [27] observed that treatment of ACE2-expressing cells with a different ER $\alpha$-glucosidase inhibitor (IHVR-17028) did not affect binding to SARS-CoV spike glycoprotein. Despite this finding, these authors reported that treated cells exhibited reduced transduction of lentiviral particles pseudotyped with SARS-CoV spike glycoprotein because of impaired SARS$\mathrm{CoV}$ spike glycoprotein-mediated membrane fusion.

Since fusion of the spike glycoprotein of coronaviruses to cellular membranes requires large conformational changes [35], we hypothesized that misfolded glycoproteins resulting from ER $\alpha$-glucosidase inhibition by miglustat could impair SARS-CoV-2 S protein-driven cell-to-cell fusion. Targeting this process seemed particularly relevant once SARS-CoV-2 has been shown to present increased fusogenic activity in comparison to SARS-CoV [36, 37] which likely facilitates infection spreading. Miglustat treatment in our in vitro model did not corroborate this assumption. Moreover, when cells from a MOGS-null patient were tested for SARS-CoV-2 Spike and ACE2 glycoprotein expression and function (i.e., syncytia formation), no differences were detected when compared to their wild-type counterparts.

Besides the SARS-CoV-2 direct cytopathic effects, immune response has shown to play a critical role in Covid-19 pathophysiology. Cytokine storm has been associated with more severe clinical symptoms and worse outcomes [38]. Interestingly, decreased proinflammatory cytokine responses have been demonstrated in an experimental model of dengue virus infection using a miglustat analog (UV-12) [39]. While our evaluation did not find miglustat having any significant effect on cytokine secretion, their function was not tested, and this could be relevant as some of those cytokines are Nglycosylated glycoproteins themselves (i.e., IL-6, MCP1) $[40,41]$.

This work was initiated based on two complementary and sequential hypotheses: (a) Covid-19-related glycoproteins (i.e., viral: SARS-CoV-2 Spike; human: ACE2) can be modified by MOGS manipulation; (b) this effect can alter Covid-19 pathophysiology with potential therapeutic use to prevent/modify/ease SARS-CoV-2 infection and its consequences. While only the first of our hypotheses was confirmed, we believe our contribution of mostly negative data is highly relevant in the current times, despite the intrinsic constraints of the study design: e.g., the experimental approach was entirely developed in vitro (as appropriate for this stage of the investigation), and the cell lines transfected with the SARS-CoV-2 Spike and ACE2 glycoprotein expression vectors (i.e., HEK293T, NIH3T3 and fibroblasts) are not the natural hosts for SARS-CoV-2 infection (e.g., respiratory tract epithelial cells). Still, only by relying on scientifically solid data, both positive as well as negative, is that progress on Covid-19 could be made [42-44]. Although somehow disappointing, our negative functional data was not a complete surprise. When altering the N-glycosylation pattern of infectious/host-related glycoproteins, the infectious diseases susceptibility balance might be unaltered, or skewed in either direction: decreased and beneficial, or increased and detrimental [1]. In our work, we show that despite clear $\mathrm{N}$-glycan alteration in the presence of miglustat, the function of the Covid-19related glycoproteins studied was not affected, making it unlikely that miglustat can change the natural course of the disease. We also do acknowledge that the methodological approach we explored was mostly focused on the early steps of the viral infection and we did not include live virus studies, limiting the impact of our findings. ${ }^{1}$ On the other hand, we did test our hypothesis on genetically null-, rather than pharmacologically inhibited-, MOGS cells and the results were not encouraging in terms of decreasing infectious susceptibility. Moreover, if the number of cells making syncytia were an indicator of increased SARS-CoV-2 virulence and viral spreading [46], MOGS deficiency/full inhibition may even be detrimental for the pathophysiology of Covid-19. Further studies are warranted to confirm this result and more importantly to find valid therapeutic options against this viral infection.

Acknowledgments This work was supported by the Intramural Research Program, National Institutes of Health Clinical Center and National Institute of Allergy and Infectious Diseases, and the NIH Clinical Center-Emergent BioSolutions Inc., M-CRADA "Antiviral activity of MOGS inhibitors." We acknowledge Drs. Kevin Spurgers, Anthony Treston, and Kelly Warfield from Emergent BioSolutions for their review of the manuscript, financial support for research of the impact of iminosugars on $\mathrm{N}$-glycosylation, and the provision of $\mathrm{N}$ butyldeoxynojirimycin. The content of this article does not necessarily reflect the views or policies of the Department of Health and Human Services, nor does mention of trade names, commercial products, or organizations implying endorsement by the US government.

Authors' Contributions SDR conceived the project; CJNS and HSK designed and executed the experiments; CJNS, HSK, and SDR provided materials and analyzed the data; CJNS and SDR wrote the manuscript; CJNS, HSK, and SDR reviewed and approved the manuscript.

Funding This work was supported by the Intramural Research Program, National Institutes of Health Clinical Center and National Institute of Allergy and Infectious Diseases, and the NIH Clinical Center-Emergent BioSolutions Inc., M-CRADA "Antiviral activity of MOGS inhibitors."

\footnotetext{
${ }^{1}$ While this manuscript was in preparation, a non-peer reviewed preprint assessing the SARS-CoV-2 antiviral activity of miglustat was released. While applying a different methodology and controls than ours (i.e., no MOGS-deficient cells were tested), the authors claim that miglustat decreased secretion of infectious virus in vitro [45].
} 


\section{Compliance with Ethical Standards}

Conflict of Interest CJNS, HSK, and SDR declare that they have no relevant conflicts of interest.

\section{References}

1. Sadat MA, Moir S, Chun TW, Lusso P, Kaplan G, Wolfe L, et al. Glycosylation, hypogammaglobulinemia, and resistance to viral infections. N Engl J Med. 2014;370(17):1615-25.

2. Alonzi DS, Scott KA, Dwek RA, Zitzmann N. Iminosugar antivirals: the therapeutic sweet spot. Biochem Soc Trans. 2017;45(2): 571-82.

3. Stanley P, Taniguchi N, Aebi M. N-glycans. In: Varki A, Cummings RD, Esko JD, Stanley P, Hart GW, Aebi M, et al., editors. Essentials of glycobiology [Internet]. 3rd ed. Cold Spring Harbor: Cold Spring Harbor Laboratory Press; 2017.

4. Fischer PB, Karlsson GB, Dwek RA, Platt FM. Nbutyldeoxynojirimycin-mediated inhibition of human immunodeficiency virus entry correlates with impaired gp120 shedding and gp41 exposure. J Virol. 1996;70(10):7153-60.

5. Zhou P, Yang XL, Wang XG, Hu B, Zhang L, Zhang W, et al. A pneumonia outbreak associated with a new coronavirus of probable bat origin. Nature. 2020;579(7798):270-3.

6. World Health Organization. Virtual press conference on COVID19 - 11 March $2020 \mathrm{https} / /$ www.who.int/docs/default-source/ coronaviruse/transcripts/who-audio-emergencies-coronaviruspress-conference-full-and-final-1 1 mar2020.pdf?sfvrsn=cb432bb3 22020

7. Huang C, Wang Y, Li X, Ren L, Zhao J, Hu Y, et al. Clinical features of patients infected with 2019 novel coronavirus in Wuhan, China. Lancet. 2020;395(10223):497-506.

8. Wang D, Hu B, Hu C, Zhu F, Liu X, Zhang J, et al. Clinical characteristics of 138 hospitalized patients with 2019 novel coronavirus-infected pneumonia in Wuhan, China. JAMA. 2020;323(11):1061-9.

9. Chu DK, Akl EA, Duda S, Solo K, Yaacoub S, Schünemann HJ, et al. Physical distancing, face masks, and eye protection to prevent person-to-person transmission of SARS-CoV-2 and COVID-19: a systematic review and meta-analysis. Lancet. 2020;395(10242): 1973-87.

10. Galea S, Merchant RM, Lurie N. The mental health consequences of COVID-19 and physical distancing: the need for prevention and early intervention. JAMA Intern Med. 2020;180:817-8.

11. Nicola M, Alsafi Z, Sohrabi C, Kerwan A, Al-Jabir A, Iosifidis C, et al. The socio-economic implications of the coronavirus pandemic (COVID-19): a review. Int J Surg. 2020;78:185-93.

12. Kartoglu UH, Moore KL, Lloyd JS. Logistical challenges for potential SARS-CoV-2 vaccine and a call to research institutions, developers and manufacturers. Vaccine. 2020;38(34):5393-5.

13. Walls AC, Park YJ, Tortorici MA, Wall A, McGuire AT, Veesler D. Structure, function, and antigenicity of the SARS-CoV-2 Spike glycoprotein. Cell. 2020;181(2):281-92.e6.

14. Watanabe Y, Allen JD, Wrapp D, McLellan JS, Crispin M. Sitespecific glycan analysis of the SARS-CoV-2 spike. Science. 2020;369(6501):330-3.

15. Coutard B, Valle C, de Lamballerie X, Canard B, Seidah NG, Decroly E. The spike glycoprotein of the new coronavirus 2019$\mathrm{nCoV}$ contains a furin-like cleavage site absent in $\mathrm{CoV}$ of the same clade. Antivir Res. 2020;176:104742.

16. Ou X, Liu Y, Lei X, Li P, Mi D, Ren L, et al. Characterization of spike glycoprotein of SARS-CoV-2 on virus entry and its immune cross-reactivity with SARS-CoV. Nat Commun. 2020;11(1):1620.
17. Chang J, Guo JT, Du Y, Block T. Iminosugar glucosidase inhibitors as broadly active anti-filovirus agents. Emerg Microbes Infect. 2013;2(11):e77.

18. Williams SJ, Goddard-Borger ED. $\alpha$-Glucosidase inhibitors as host-directed antiviral agents with potential for the treatment of COVID-19. Biochem Soc Trans. 2020;48(3):1287-95.

19. Yan R, Zhang Y, Li Y, Xia L, Guo Y, Zhou Q. Structural basis for the recognition of SARS-CoV-2 by full-length human ACE2. Science. 2020;367(6485):1444-8.

20. Fischl MA, Resnick L, Coombs R, Kremer AB, Pottage JC, Fass RJ, et al. The safety and efficacy of combination N-butyldeoxynojirimycin (SC-48334) and zidovudine in patients with HIV-1 infection and 200-500 CD4 cells/mm3. J Acquir Immune Defic Syndr (1988). 1994;7(2):139-47.

21. Patterson MC, Vecchio D, Prady H, Abel L, Wraith JE. Miglustat for treatment of Niemann-Pick C disease: a randomised controlled study. Lancet Neurol. 2007;6(9):765-72.

22. Cox TM, Aerts JM, Andria G, Beck M, Belmatoug N, Bembi B, et al. The role of the iminosugar N-butyldeoxynojirimycin (miglustat) in the management of type I (non-neuronopathic) Gaucher disease: a position statement. J Inherit Metab Dis. 2003;26(6):513-26.

23. Zhao P, Praissman JL, Grant OC, Cai Y, Xiao T, Rosenbalm KE, et al. Virus-receptor interactions of glycosylated SARS-CoV-2 Spike and human ACE2 receptor. bioRxiv. 2020.

24. Shajahan A, Archer-Hartmann S, Supekar NT, Gleinich AS, Heiss $\mathrm{C}$, Azadi P. Comprehensive characterization of $\mathrm{N}-$ and $\mathrm{O}-$ glycosylation of SARS-CoV-2 human receptor angiotensin converting enzyme 2. bioRxiv. 2020. https://doi.org/10.1101/ 2020.05.01.071688.

25. Brand M, Muller A, Alsop J, van Schaik IN, Bembi B, Hughes D. Results from a 9-year intensive safety surveillance scheme (IS(3) ) in miglustat (Zavesca(®) ) -treated patients. Pharmacoepidemiol Drug Saf. 2015;24(3):329-33.

26. Fukushi M, Yoshinaka Y, Matsuoka Y, Hatakeyama S, Ishizaka Y, Kirikae T, et al. Monitoring of S protein maturation in the endoplasmic reticulum by calnexin is important for the infectivity of severe acute respiratory syndrome coronavirus. J Virol. 2012;86(21):11745-53.

27. Zhao X, Guo F, Comunale MA, Mehta A, Sehgal M, Jain P, et al. Inhibition of endoplasmic reticulum-resident glucosidases impairs severe acute respiratory syndrome coronavirus and human coronavirus NL63 spike protein-mediated entry by altering the glycan processing of angiotensin I-converting enzyme 2. Antimicrob Agents Chemother. 2015;59(1):206-16.

28. Wan Y, Shang J, Graham R, Baric RS, Li F. Receptor recognition by the novel coronavirus from Wuhan: an analysis based on decade-long structural studies of SARS coronavirus. J Virol. 2020;94(7): e00127-20.

29. Wang Q, Zhang Y, Wu L, Niu S, Song C, Zhang Z, et al. Structural and functional basis of SARS-CoV-2 entry by using human ACE2. Cell. 2020;181(4):894-904.e9.

30. Wrapp D, Wang N, Corbett KS, Goldsmith JA, Hsieh CL, Abiona O, et al. Cryo-EM structure of the 2019-nCoV spike in the prefusion conformation. Science. 2020;367(6483):1260-3.

31. Lan J, Ge J, Yu J, Shan S, Zhou H, Fan S, et al. Structure of the SARS-CoV-2 spike receptor-binding domain bound to the ACE2 receptor. Nature. 2020;581(7807):215-20.

32. Li Q, Wu J, Nie J, Zhang L, Hao H, Liu S, et al. The impact of mutations in SARS-CoV-2 spike on viral infectivity and antigenicity. Cell. 2020;182(5):1284-94.e9.

33. Mehdipour AR, Hummer G. Dual nature of human ACE2 glycosylation in binding to SARS-CoV-2 spike. bioRxiv. 2020. https:// doi.org/10.1101/2020.07.09.193680.

34. Procko E. The sequence of human ACE2 is suboptimal for binding the S spike protein of SARS coronavirus 2. bioRxiv. 2020. 
35. Walls AC, Tortorici MA, Snijder J, Xiong X, Bosch BJ, Rey FA, et al. Tectonic conformational changes of a coronavirus spike glycoprotein promote membrane fusion. Proc Natl Acad Sci U S A. 2017;114(42):11157-62.

36. Zhu Y, Yu D, Yan H, Chong H, He Y. Design of potent membrane fusion inhibitors against SARS-CoV-2, an emerging coronavirus with high fusogenic activity. J Virol. 2020;94(14):e00635-20.

37. Xia S, Liu M, Wang C, Xu W, Lan Q, Feng S, et al. Inhibition of SARS-CoV-2 (previously 2019-nCoV) infection by a highly potent pan-coronavirus fusion inhibitor targeting its spike protein that harbors a high capacity to mediate membrane fusion. Cell Res. 2020;30(4):343-55.

38. Pedersen SF, Ho YC. SARS-CoV-2: a storm is raging. J Clin Invest. 2020;130(5):2202-5.

39. Warfield KL, Plummer E, Alonzi DS, Wolfe GW, Sampath A, Nguyen T, et al. A novel iminosugar UV-12 with activity against the diverse viruses influenza and dengue (novel iminosugar antiviral for influenza and dengue). Viruses. 2015;7(5):2404-27.

40. Riethmueller S, Somasundaram P, Ehlers JC, Hung CW, Flynn CM, Lokau J, et al. Proteolytic origin of the soluble human IL-6R in vivo and a decisive role of N-glycosylation. PLoS Biol. 2017;15(1):e2000080.

41. Proost P, Struyf S, Couvreur M, Lenaerts JP, Conings R, Menten P, et al. Posttranslational modifications affect the activity of the human monocyte chemotactic proteins MCP-1 and MCP-2: identification of MCP-2(6-76) as a natural chemokine inhibitor. J Immunol. 1998;160(8):4034-41.

42. Cavalcanti AB, Zampieri FG, Rosa RG, Azevedo LCP, Veiga VC, Avezum A, et al. Hydroxychloroquine with or without azithromycin in mild-to-moderate covid-19. N Engl J Med. 2020;383(21):2041-52.

43. Mehra MR, Desai SS, Kuy S, Henry TD, Patel AN. Retraction: cardiovascular disease, drug therapy, and mortality in Covid-19. N Engl J Med. DOI: 10.1056/NEJMoa2007621. N Engl J Med. 2020;382(26):2582.

44. Mehra MR, Ruschitzka F, Patel AN. Retractionhydroxychloroquine or chloroquine with or without a macrolide for treatment of COVID-19: a multinational registry analysis. Lancet. 2020;395(10240):1820.

45. Rajasekharan S, Bonotto RM, Kazungu Y, Alves LN, Poggianella M, Orellana PM, et al. Repurposing of miglustat to inhibit the coronavirus severe acquired respiratory syndrome SARS-CoV-2. bioRxiv. 2020. https://doi.org/10.1101/2020.05.18.101691.

46. Zhong P, Agosto LM, Munro JB, Mothes W. Cell-to-cell transmission of viruses. Curr Opin Virol. 2013;3(1):44-50.

Publisher's Note Springer Nature remains neutral with regard to jurisdictional claims in published maps and institutional affiliations. 